\title{
Herramienta de evaluación continua basada en problemas personalizados de corrección automática: análisis de su implantación en la asignatura "Mercados Energéticos"
}

\section{Manuel Alcázar-Ortega ${ }^{\mathrm{a}}$, Carlos Álvarez-Bel ${ }^{\mathrm{a}}$}

a Departamento de Ingeniería Eléctrica, Universidad Politécnica de Valencia. Camino de Vera, s/n, edificio 5E, 2º Planta.46022 Valencia (España). malcazar@iie.upv.es, calvarez@die.upv.es.

\begin{abstract}
The strategies for learning of technical teaching are based, to a large extent, on the realization of practical problems on the part of the students. However, the correct use of these strategies would entail a personalized correction and a follow-up that, when the volume of students is high, is practically unmanageable. In this context, this work presents a tool that facilitates the realization of personalized problems for each student, its semi-automatic correction and the feedback to the student in order to facilitate their learning and to carry out a continuous follow-up throughout the course. This tool has been successfully implemented into the course on "Energy Markets", corresponding to the Degree in Energy Engineering of the Polytechnic University of Valencia. Moreover, the impact on the obtained results obtained by students in the last years is analyzed in this work.
\end{abstract}

Keywords: tool, problems, self-evaluation, feedback, engineering, energy, markets

\begin{abstract}
Resumen
Las estrategias de aprendizaje de las enseñanzas técnicas están basadas, en gran medida, en la realización de problemas prácticos por parte de los alumnos. Sin embargo, la correcta utilización de estas estrategias conllevaría una corrección personalizada y un seguimiento que, cuando el volumen de alumnos es elevado, resulta prácticamente ingestionable. En este contexto, este trabajo presenta una herramienta que facilita la realización de problemas personalizados para cada estudiante, su corrección semiautomática y la realimentación al alumno de cara a facilitar su aprendizaje y realizar un seguimiento continuo a lo largo del curso. Esta herramienta se ha implantado con éxito en la asignatura "Mercados Energéticos", correspondiente al Grado en Ingeniería de la Energía de la Universidad Politécnica de Valencia, cuyo impacto en los resultados obtenidos por los estudiantes en los últimos años se analiza en este trabajo.
\end{abstract}

Palabras clave: herramienta, problemas, autoevaluación, realimentación, ingeniería, energía, mercados 
Herramienta de evaluación continua basada en problemas personalizados de corrección automática: análisis de su implantación en la asignatura "Mercados Energéticos"

\section{Introducción}

El desarrollo de herramientas tecnológicas (informáticas o de otro tipo) para facilitar el aprendizaje de los estudiantes de ingeniería se ha demostrado muy útil a la hora de hacerles más atractivo este campo (Plaza Merino et al, 2016), proporcionando a los alumnos una mejor comprensión de la materia y a los profesores un medio para impartir las clases de una forma más estimulante (Carreras y Snider, 1998).

Entre otras ventajas, las herramientas tecnológicas favorecen la realimentación a los alumnos sobre sus aciertos y errores, lo que conlleva un proceso de aprendizaje más consistente (Jattie y Timperley, 2007). Sin embargo, dado el elevado número de estudiantes o la excesiva dedicación necesaria para la corrección de ejercicios, no es viable realizarla en un tiempo adecuado, lo que constituye un problema importante (Wiggins, 2012). En este sentido, la herramienta aquí propuesta ataca directamente este problema, proporcionando una corrección suficientemente rápida y una comunicación con los alumnos casi automática, a través del correo electrónico.

Existen herramientas desarrolladas por docentes para favorecer la comprensión de algunos temas específicos dentro del campo de la ingeniería, tales como los sistemas eléctricos de potencia (Córdova-Acosta et al, 2018) o ingeniería de procesos (Wagner et al, 2016). La herramienta desarrollada en el trabajo que aquí se presenta se centra en aspectos relacionados con los mercados energéticos, cuya trascendencia es cada vez más importante en las enseñanzas técnicas universitarias.

La producción, suministro y compra-venta de energía ha tenido tradicionalmente una estructura muy simple, donde un mismo agente realizaba, de forma monopolista y verticalmente integrada, todas las actividades relacionadas. Sin embargo, debido a las múltiples ineficiencias en este modo de funcionar (que resultaba en precios excesivos para los consumidores de productos energéticos), la situación cambió drásticamente a final del siglo XX, dando como resultado un modelo de mercado liberalizado mediante la creación de diferentes estructuras complejas para desarrollar las actividades comerciales y técnicas relacionadas con la energía (Strbac y Kirschen, 2004). Estas estructuras incluyen mercados, operadores, complejas redes de suministro, etc., donde intervienen múltiples agentes realizando labores especializadas cada uno y que requieren en algunos casos una coordinación muy precisa.

La comprensión de todos estos aspectos no es sencilla, sobre todo para el alumno que se enfrenta por primera vez a esta problemática. Por lo tanto, el desarrollo de este tipo de herramientas, que de forma interactiva le permitirán adquirir soltura en la gestión de estos conceptos, resulta esencial para llevar a cabo un aprendizaje continuo y donde los conocimientos básicos utilizados en este campo creen un sustrato consistente que le servirá a lo largo de toda su vida académica y profesional.

El artículo se estructura de la siguiente forma: El capítulo 2 presenta los objetivos de la herramienta, los cuales se desarrollarán de forma detallada en el capítulo 3, donde se describen sus funcionalidades y principales características. El capítulo 4 muestra los resultados obtenidos a lo largo de los últimos años en la asignatura de Mercados

(cc) EY-NC-ND 2018, Universitat Politècnica de València

Congreso IN-RED (2018) 
Energéticos, donde la herramienta se ha implantado durante tres cursos, permitiendo evaluar el impacto que su utilización ha supuesto en los alumnos. Finalmente, las conclusiones del presente trabajo se incluyen en el capítulo 5.

\section{Objetivos}

Los objetivos en base a los cuales se ha diseñado y aplicado esta herramienta son los siguientes:

- En primer lugar, permitir que los alumnos pongan en práctica los conocimientos adquiridos en clase a lo largo del curso a través de problemas directamente relacionados con los contenidos de las diferentes unidades temáticas.

- Conseguir que los alumnos pongan en práctica dichos conocimientos de forma individualizada, ya que los problemas están particularizados para cada estudiante en función de su número de identificación personal (DNI o NIE)

- Favorecer el trabajo en grupo, dado que, aunque los datos de los ejercicios son diferentes para cada alumno, los problemas propuestos pueden ser abordados de forma conjunta por varios alumnos. De esta manera, grupos de estudiantes colaborarían para obtener el procedimiento de resolución, aunque cada uno de ellos tuviera que trabajar de forma individual con sus datos personales para obtener el resultado definitivo.

- Permitir al profesor realizar un seguimiento particularizado de cada alumno de una manera cómoda, rápida y efectiva, ya que los problemas se corrigen automáticamente a través de la herramienta.

- Finalmente, ayudar a que el alumno pueda auto-evaluar su proceso de aprendizaje de forma progresiva al recibir por correo electrónico la corrección de sus ejercicios, permitiéndole, de esta forma, aprender de sus errores y afianzar los conocimientos adquiridos.

\section{Desarrollo de la innovación}

La herramienta se ha programado utilizando el paquete Visual Basic proporcionado por Microsoft EXCEL. De esta forma, los problemas a resolver se facilitan a los alumnos en formato .xls, lo que hace posible que puedan ser utilizados en cualquier ordenador personal.

La herramienta consta de dos módulos:

- Una plantilla que se entrega a los alumnos con los enunciados de los problemas que tienen que resolver y los espacios necesarios para indicar las respuestas.

- Un corrector donde el profesor puede cargar la información proporcionada por los alumnos en cada una de las plantillas. Dicho corrector permite no sólo evaluar los resultados de cada ejercicio, sino que también es capaz de enviar de forma automática un correo electrónico a cada estudiante informándole de su 
Herramienta de evaluación continua basada en problemas personalizados de corrección automática: análisis de su implantación en la asignatura "Mercados Energéticos"

calificación, de las respuestas correctas o incorrectas y de la solución adecuada en el caso de las incorrectas.

\subsection{Plantilla para el alumno}

En la plantilla que se facilita al alumno están los enunciados de los problemas a resolver. Normalmente, cada plantilla contiene entre 2 y 3 ejercicios, cada uno de los cuales se estructura en diversos apartados.

La información que el alumno debe introducir, tal y como se muestra en la Figura 1, es la siguiente:

- Su nombre y apellidos

- $\quad$ Su número de identificación, sin letras (DNI, NIE, pasaporte). Esta información es importante porque en base a ella se calcularán los coeficientes con los que se obtienen los parámetros de los ejercicios a resolver.

- Su e-mail. Esta dirección de correo se utilizará para notificar al alumno su calificación y el resultado de la corrección de la plantilla.

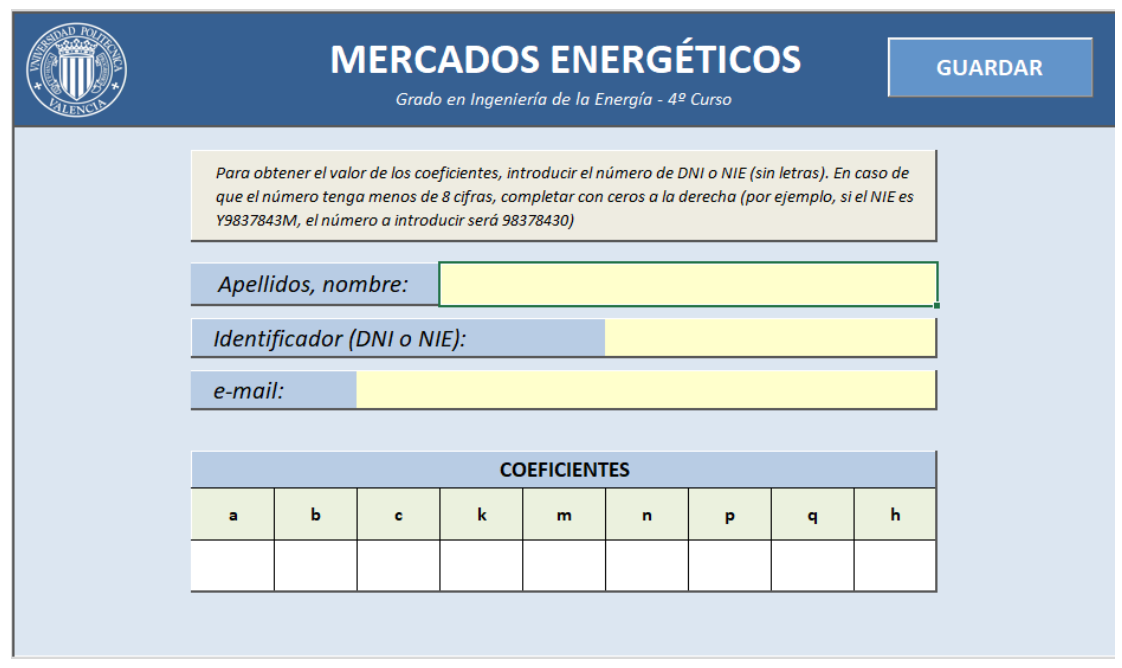

Figura 1. Plantilla para la introducción de datos del alumno

\subsubsection{Cálculo de los coeficientes}

Los coeficientes en base a los cuales se obtendrán los parámetros de los ejercicios están calculados a partir de los caracteres del número de identificación del alumno. Para realizar dicho cálculo, es necesario que el número de identificación del alumno tenga exactamente 8 cifras. En caso de que el número de identificación tuviera letras o un número de cifras diferente a 8, el alumno tendría que escribir únicamente las 8 primeras cifras, o completar a 
la derecha con cerros hasta alcanzar el número indicado. Al hacerlo, la plantilla calcularía los coeficientes, tal y como se muestra en la Figura 2.

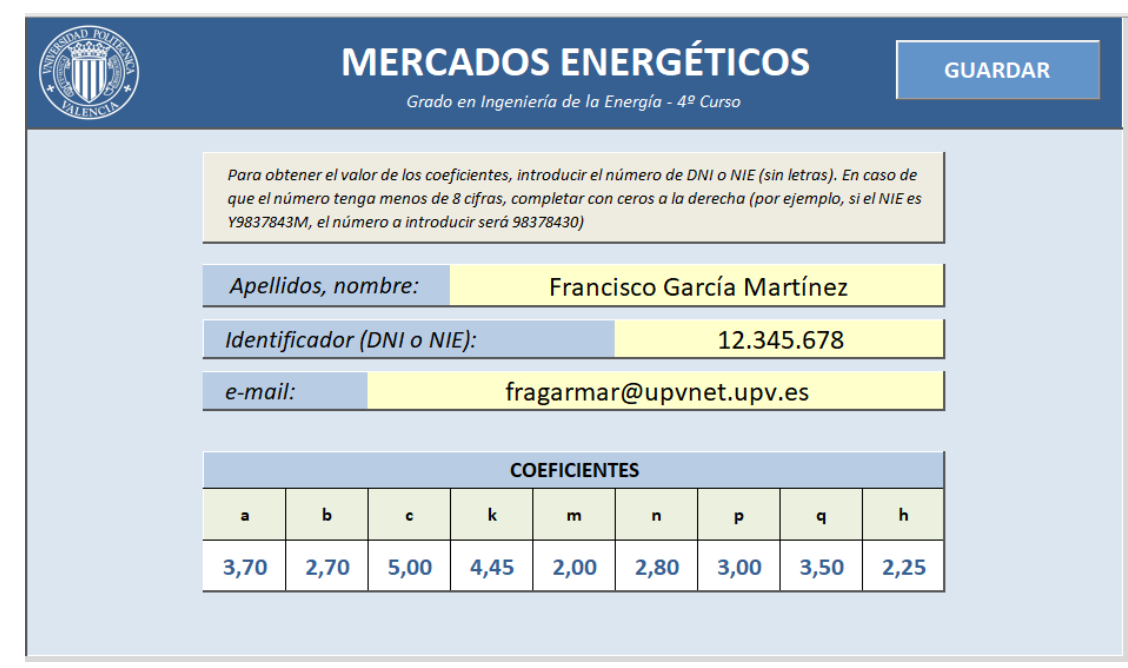

Figura 2. Obtención de los coeficientes de cálculo

En función de sus 8 caracteres, el número de identificación $I D$ del alumno que utiliza la herramienta sería el siguiente:

$$
I D=\sum_{i=1}^{8} N_{i} \cdot 10^{(8-i)}
$$

Donde $\mathrm{N}_{1}, \mathrm{~N}_{2}, \ldots \mathrm{N}_{8}$ son cada uno de los caracteres del número de identificación del alumno (unidades, decenas, centenas,...).

Utilizando estos caracteres, la herramienta calcula los 9 coeficientes para la parametrización de los problemas. Estos coeficientes se ajustan de manera semi-empírica, de forma que los parámetros de los enunciados tengan una variedad admisible y los resultados obtenidos sean razonables. Como ejemplo, se muestran las expresiones utilizadas para calcular los coeficientes $a$ y $b$, siendo análogo el cálculo del resto de coeficientes: 


$$
\begin{aligned}
& a=\left\{\begin{array}{l}
1, \text { si }\left(\frac{N_{4}}{1,5}+1\right) \leq 0 \\
\left(\frac{N_{4}}{1,5}+2\right), \text { si } 0<\left(\frac{N_{4}}{1,5}+1\right)<1 \\
\left(\frac{N_{4}}{1,5}+1\right), \text { si } 1 \leq\left(\frac{N_{4}}{1,5}+1\right)<4 \\
\left(\frac{N_{4}}{3}+\frac{1}{2}\right), \text { si }\left(\frac{N_{4}}{1,5}+1\right) \geq 4
\end{array}\right. \\
& b=\left\{\begin{array}{l}
1, \text { si }\left(N_{5}+N_{2}-\frac{N_{3}}{5}\right) \leq 0 \\
\left(N_{5}+N_{2}-\frac{N_{3}}{5}\right)+1, \text { si } 0<\left(N_{5}+N_{2}-\frac{N_{3}}{5}\right)<1 \\
\left(N_{5}+N_{2}-\frac{N_{3}}{5}\right), \text { si } 1 \leq\left(N_{5}+N_{2}-\frac{N_{3}}{5}\right) \leq 4 \\
\left(\frac{N_{5}+N_{2}}{2}-\frac{N_{3}}{10}\right), \text { si }\left(N_{5}+N_{2}-\frac{N_{3}}{5}\right)>4
\end{array}\right.
\end{aligned}
$$

\subsubsection{Diseño del enunciado e identificación de las variables}

La Figura 3 muestra el enunciado de uno de los problemas propuestos en la asignatura de Mercados Energéticos, correspondiente al tema "Introducción a los mercados energéticos: conceptos de microeconomía”. El diseño e implementación del enunciado de cada problema debe considerar los siguientes pasos:

- En primer lugar, se debe elegir un problema que pueda ser resuelto de forma automática, por lo que la respuesta a las preguntas realizadas debe ser cerrada y, preferiblemente, numérica. En caso de no ser numérica, las posibles respuestas deben estar previamente establecidas de cada a no ofrecer duda al alumno a la hora de responder.

- Es necesario identificar los parámetros que se van a personalizar para cada alumno en función de los coeficientes calculados en el apartado anterior. En el caso del ejemplo mostrado en la Figura 3, los parámetros seleccionados aparecen remarcados en color azul y están calculados a partir de los coeficientes obtenidos a partir del número de identificación del alumno. Por ejemplo, en la primera ecuación del problema, parametrizada de la siguiente manera:

$$
C(y)=A \cdot y^{2}+B \cdot y+C
$$


los factores A, B y C se calcularían de la siguiente forma:

$$
\begin{gathered}
A=10 \cdot k \\
B=100 \cdot m \\
C=10000 \cdot n
\end{gathered}
$$

donde k, m y n son los coeficiente mostrados en la Figura 2 que se calculan en función del número de identificación del alumno.

- Finalmente, hay que indicar el lugar en el que el alumno debe contestar la pregunta considerada en el enunciado. La respuesta a cada pregunta debe ser única y el lugar donde debe ser contestada debe estar claramente definido. Es esencial que estén indicadas las unidades en las que la pregunta debe ser contestada, ya que, de otra forma, la herramienta no interpretaría correctamente el resultado. En el ejemplo de la Figura 3 se indica con un cuadro de color amarillo el lugar donde cada respuesta debe ser consignada.

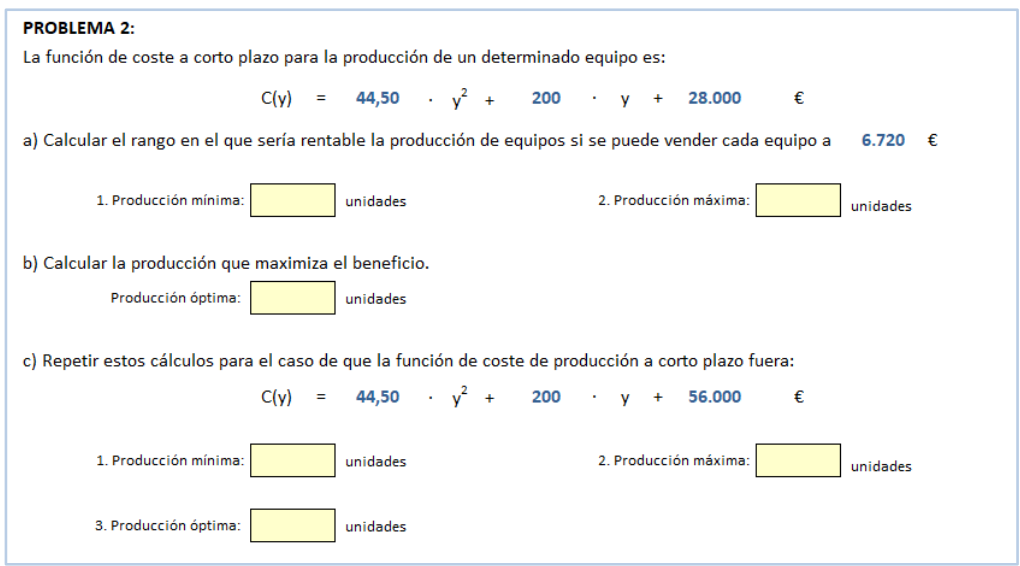

Figura 3. Diseño del enunciado e identificación de variables (azul)

Es posible que, en función de los parámetros de un alumno en concreto, alguno de los apartados no tenga solución. En ese caso, se debe indicar en las instrucciones que se facilitan cómo ha de proceder el alumno, ya que no es lo mismo no responder una pregunta, que el que la solución sea cero o no exista. En la herramienta, esta casuística se ha resuelto de la siguiente manera:

- Si la celda de una respuesta está vacía, se considera que el alumno no ha sido capaz de obtener la respuesta y no la ha contestado.

- Si la celda contiene un cero, significa que la respuesta a la pregunta es igual a cero.

- Si, debido a los parámetros particulares del alumno, la respuesta no tiene solución, se deberá indicar en la celda $N / E$ (no existe).

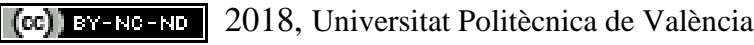

Congreso In-Red (2018) 
Herramienta de evaluación continua basada en problemas personalizados de corrección automática: análisis de su implantación en la asignatura "Mercados Energéticos"

\subsubsection{Diseño del enunciado e identificación de las variables}

Cuando la plantilla ha sido completada por el alumno, éste debe guardarla y puede enviarla para su corrección al profesor indicado, bien por correo electrónico o mediante otras plataformas habilitadas al efecto (por ejemplo, Poliformat en la UPV). Para ello puede hacer uso de un botón de "Guardar" habilitado para tal fin, tal y como se muestra en el ángulo superior derecho de la Figura 2.

\subsection{Corrector para el profesor}

El proceso de corrección de los ejercicios planteados en la plantilla descrita en el apartado anterior debe ser rápido y automático. Para ello, la herramienta dispone de un corrector que, de forma secuencial, realiza las siguientes tareas, tal y como se muestra en la Figura 4 :

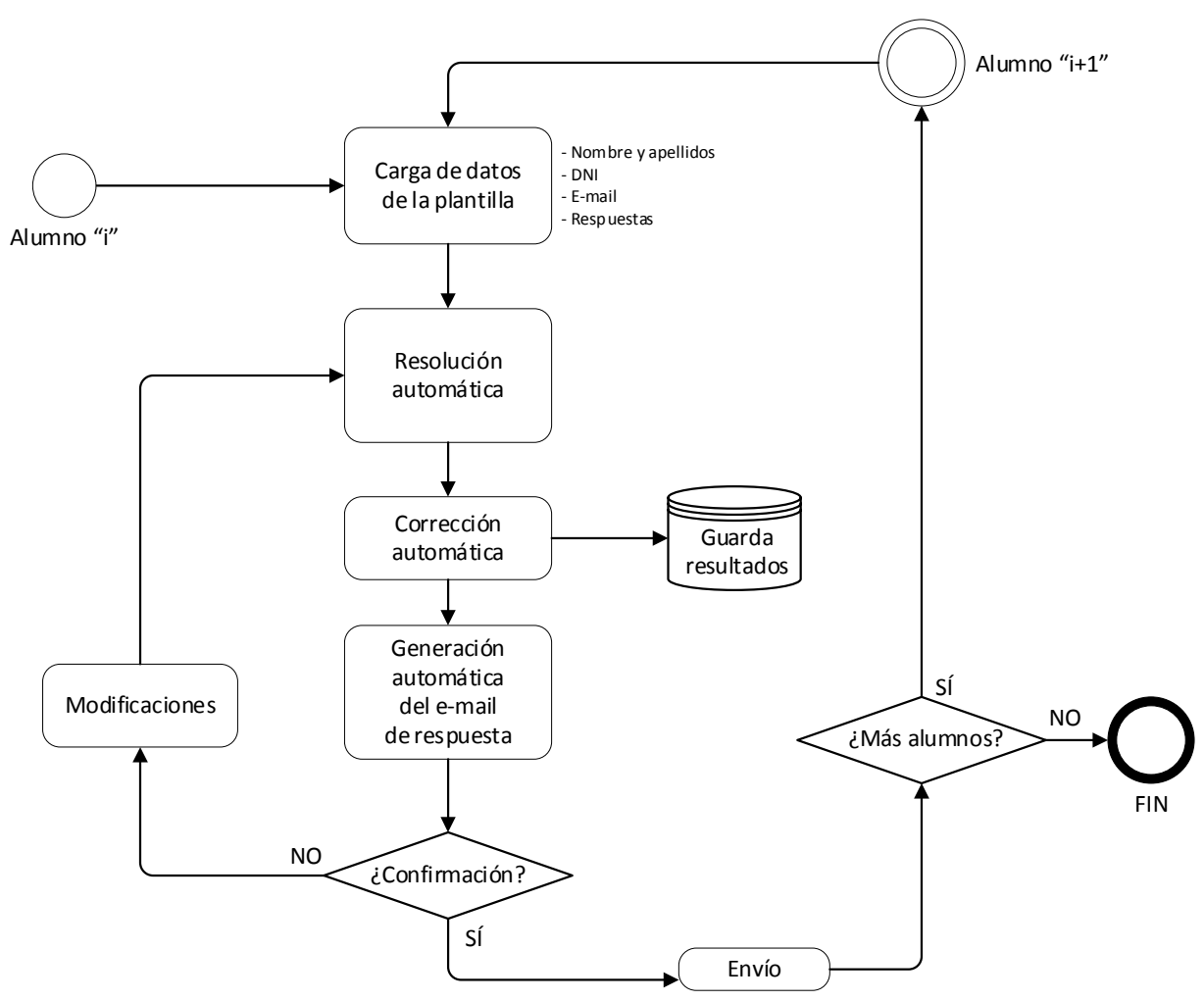

Figura 4. Secuencia de tareas de la herramienta de corrección

1. Carga de los datos de la plantilla. Esta acción se ejecuta pulsando un botón que permite seleccionar el fichero donde se encuentra la plantilla del alumno deseado. Los datos que se cargan corresponden a la identificación del alumno (nombre,

(cc) EY-NC-ND 2018, Universitat Politècnica de València

Congreso IN-RED (2018) 
apellidos, DNI y correo electrónico) y a las respuestas que haya proporcionado en la plantilla a evaluar.

2. Resolución automática. El corrector resuelve automáticamente los ejercicios planteados en función de los coeficientes del alumno, calculados a partir de su número de identificación personal.

3. Corrección automática. El corrector compara los datos cargados de la plantilla del alumno con los resultados correctos evaluados en el paso anterior y determina qué respuestas son correctas y qué respuestas son erróneas. De cara a evitar el efecto de los posibles redondeos a la hora de considerar un número determinado de cifras significativas, el corrector tiene un margen de tolerancia personalizable. Por defecto, se ha establecido el error máximo admisible en un $\pm 5 \%$. Una vez realizada la corrección, la herramienta guarda el resultado en la lista de calificaciones de la asignatura.

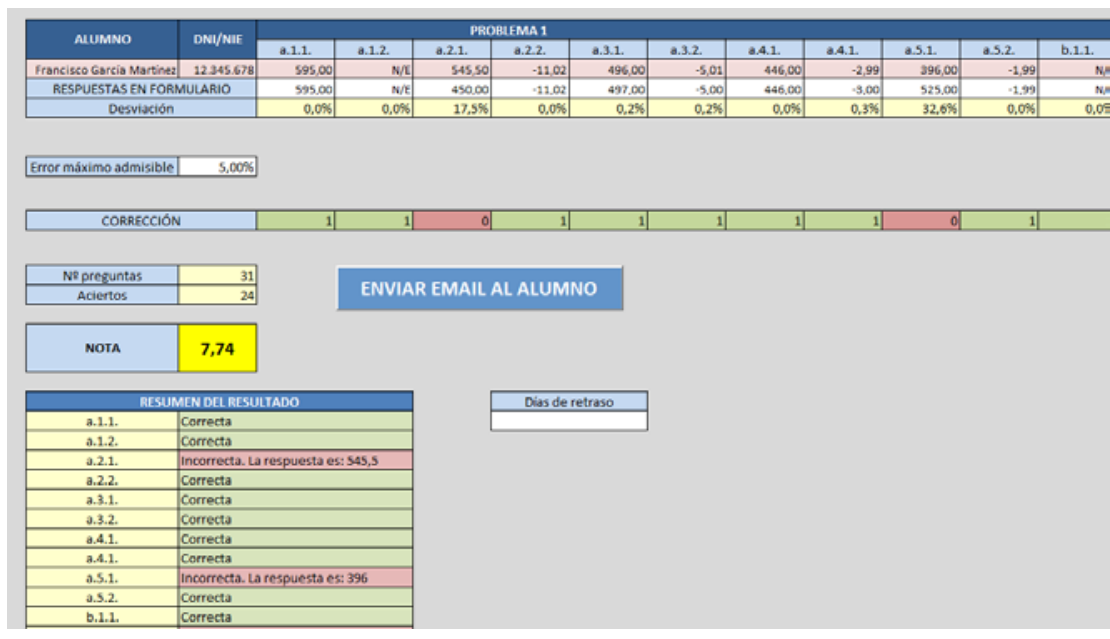

Figura 5. Fragmento de la pantalla de la herramienta de corrección

4. Generación automática del e-mail de respuesta. De cara a notificar al alumno sobre el resultado de su ejercicio, la herramienta dispone de un generador automático de correos electrónicos, el cual enviará un e-mail a cada alumno indicando:

- Su calificación en el ejercicio entregable, que se calculará en función del número de aciertos y de errores.

- Qué preguntas son correctas y cuáles incorrectas.

- $\quad$ En el caso de las preguntas incorrectas, cuál es la solución correcta.

Un ejemplo del tipo de e-mail enviado se muestra en la Figura 6.

5. Confirmación de envío. Una vez generado el correo automático, el profesor debe confirmar si desea enviarlo o si, por el contrario, es necesario realizar alguna 
Herramienta de evaluación continua basada en problemas personalizados de corrección automática: análisis de su implantación en la asignatura "Mercados Energéticos"

revisión o modificación sobre la corrección. Si el profesor confirma el envío, la corrección del alumno considerado habrá finalizado y la herramienta permitirá entonces cargar la plantilla del siguiente alumno, comenzando nuevamente el proceso.

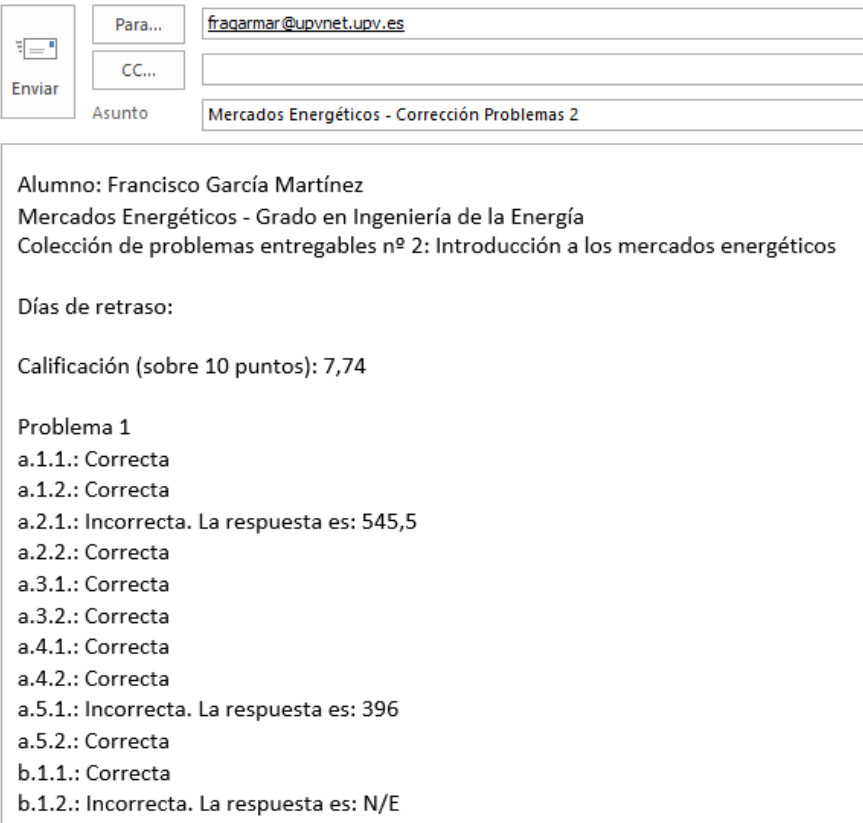

Figura 6. Ejemplo de correo electrónico sobre la corrección del ejercicio entregable

Por último, el corrector permite aplicar una penalización a los ejercicios que hayan sido entregados fuera de la fecha indicada. Por ejemplo, para el caso de aplicación considerado en este artículo, cuyos resultados se presentan la sección siguiente, se dio a los alumnos un plazo de 10 días para completar la resolución del ejercicio desde la publicación del enunciado, aplicándose una penalización de 0,1 puntos por día de retraso para las entregas fuera de plazo.

\section{Resultados: impacto en las calificaciones de los alumnos}

La herramienta descrita en la sección anterior se ha utilizado en varias asignaturas impartidas por los autores en la Universidad Politécnica de Valencia. En particular, se presenta en este apartado el análisis comparativo de los resultados obtenidos por los alumnos en la asignatura "Mercados Energéticos", correspondiente a la titulación de Grado en Ingeniería Energética que se imparte en la Escuela Técnica Superior de Ingenieros Industriales, antes y después de aplicarse dicha herramienta. De esta forma, puede medirse de una forma objetiva el impacto producido por su implantación mediante el análisis de las calificaciones históricas en dicha asignatura durante un período de 8 años. Los resultados se

(c) EY-NC-ND 2018, Universitat Politècnica de València

Congreso IN-RED (2018) 
han organizado en 20 clases, considerándose la puntuación obtenida por los alumnos de 0 a 10 con un intervalo de 0,5 puntos.
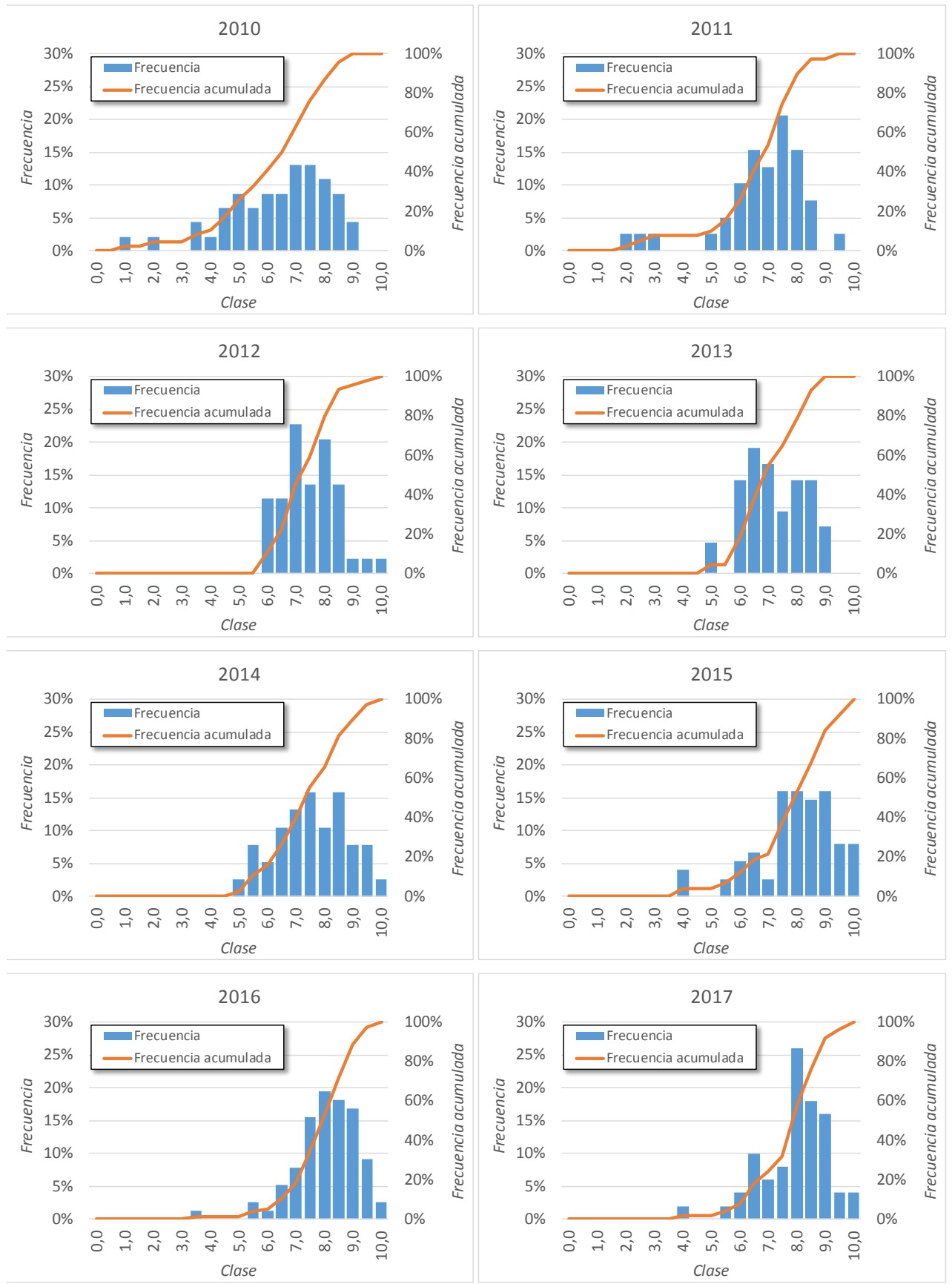

Figura 7. Histogramas de calificación de la asignatura "Mercados Energéticos" 
Herramienta de evaluación continua basada en problemas personalizados de corrección automática: análisis de su implantación en la asignatura "Mercados Energéticos”

Hasta el curso 2012-2013, dicha asignatura formaba parte del plan de estudios del Máster en Tecnología Energética para un Desarrollo Sostenible. Desde el curso 2013-2014, la asignatura de trasladó a la titulación del Grado. Sin embargo, como puede apreciarse en los histogramas de las calificaciones de los alumnos mostrados en la Figura 7, los cambios comienzan a apreciarse a partir del curso 2015-2016, que es el momento en que se implanta la herramienta.

En los años anteriores a 2015, el $40 \%$ de los alumnos obtuvo calificaciones de 7 puntos (sobre un máximo de 10 puntos) o menos. En cambio, la franja de alumnos con esta puntuación se redujo a la mitad a partir de 2015, cuando se implantó la herramienta, pasando al 21\%. Esto quiere decir que el $79 \%$ de los alumnos obtuvo más de 7 puntos como nota media, lo que supone un incremento de 20 puntos porcentuales con respecto a los resultados de años anteriores. Estos porcentajes se han mantenido durante los dos años siguientes en los que la herramienta ha continuado utilizándose, con un 82\% (2016) y un 76\% (2017) de alumnos que han superado los 7 puntos en su nota media.

Este resultado también se aprecia en la parte superior de la escala de evaluación, ya que si hasta el año 2014 el número de alumnos que alcanzó la calificación de sobresaliente (9 puntos) se situaba en un 7-8\%, a partir del año 2015 esta proporción ha llegado al 16\% (2015), 17\% (2016) y 16\% (2017), lo que supone un incremento del $100 \%$ con respecto a los resultados obtenidos antes de implantarse la herramienta.

Estos resultados se muestran gráficamente de una forma más evidente en la Figura 8, donde se representa el diagrama de porcentaje acumulado para todos los años evaluados.

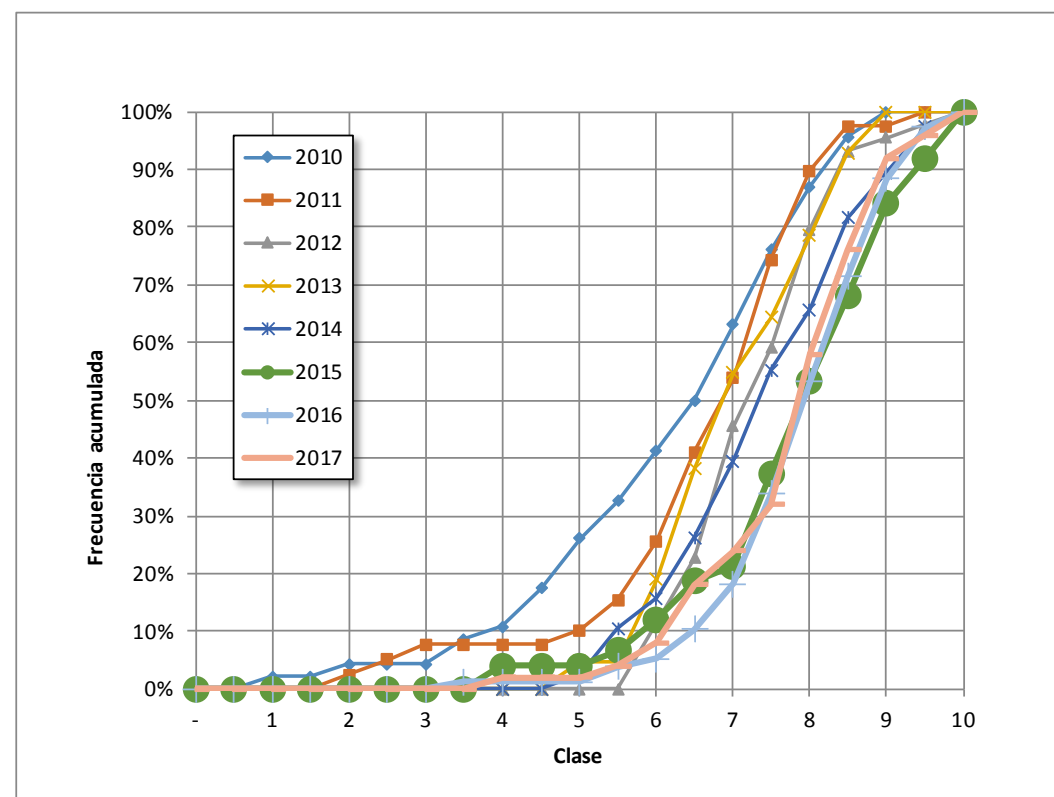

Figura 8. Frecuencia acumulada de calificaciones. 2010 - 2017

(cc) EY-NC-ND 2018, Universitat Politècnica de València 
Tal y como se aprecia en la figura, las series correspondientes a los tres últimos años aparecen claramente desplazadas hacia la derecha, lo que denota una mejora sustancial en las calificaciones obtenidas por los alumnos tras la utilización de la herramienta.

\section{Conclusiones}

Este artículo presenta una herramienta de evaluación continua, basada en la realización de ejercicios prácticos en el campo de la ingeniería, que permite realizar una corrección automática y una rápida realimentación a los alumnos que les permita mejorar en su proceso de aprendizaje. La herramienta se basa en una plantilla facilitada al estudiante y un corrector para el uso del profesor. Se presenta un caso práctico de aplicación donde la herramienta, programada en Microsoft EXCEL, se ha implantado con éxito en la asignatura "Mercados Energéticos" que se imparte en la Universidad Politécnica de Valencia. El impacto en las calificaciones de los estudiantes se observa desde el primer año de aplicación, mejorando tanto en la calificación media como la proporción de estudiantes con resultados más altos. De este modo, la proporción de estudiantes con al menos 7 puntos sobre 10 se ha incrementado en 20 puntos porcentuales, mientras que el número de alumnos con calificación de sobresaliente se ha duplicado, alcanzando el 16\% en el último curso. Estos resultados evidencian la conveniencia de desarrollar y utilizar herramientas como la que aquí se presenta para facilitar el seguimiento continuo del proceso de aprendizaje de los alumnos, mejorar sus resultados académicos y favorecer el desarrollo de competencias profesionales.

\section{Referencias}

CARRERAS, F. y SNIDER, A.D. (1998). "A Matlab educational software tool for teaching complex analysis in engineering" en IEEE Southeastcon '98: Engineering for a New Era (24-26 abril 1998). Orlando, FL, USA: IEEE, p. 204-207. DOI: 10.1109/SECON.1998.673329

CÓRDOVA-ACOSTA, A., CALDERÓN-GUIZAR, J.G., FRAGOSO-DÍAZ, O.G. (2018). "An educational tool for introducing power engineering students with modal extraction concepts". Computer Applications in Engineering Education, p. 1-13. DOI: 10.1002/cae.21899

HATTIE, J. y TIMPERLEY, H. (2007). "The power of feedback" en Review of Educational Research, vol. 77, issue 1, p. 81-112. DOI: 10.3102/003465430298487

PLAZA MERINO, P., SANCRISTÓBAL RUIZ, E., CARRO FERNÁNDEZ, G. y CASTRO GIL, M. (2016). "Robotic Educational Tool to engage students on Engineering" en IEEE Frontiers in Education Conference (12-15 octubre 2016). Erie, PA, USA: IEEE, 1-4. DOI: 10.1109/FIE.2016.7757417

STRBAC, G. y KIRSCHEN, D. (2004). Fundamentals of Power System Economics. John Wiley \& Sons, Ltd. ISBN: 978-0-470-02058-6 
Herramienta de evaluación continua basada en problemas personalizados de corrección automática: análisis de su implantación en la asignatura "Mercados Energéticos"

WAGNER, C., SCHÜLlER, A., FLEISCHACKER, C. y EPPLE, U. (2016). "An Educational Framework for Process Control Theory and Engineering Tools" en $11^{\text {th }}$ IFAC Symposium on Advances in Control Education (1-3 junio 2016). Bratislava, República Eslovaca. IFAC-PapersOnLine, vol. 49, issue 6, p. 46-51. DOI: 10.1016/j.ifacol.2016.07.151

WIGGINS, G. (2012). "Seven keys to effective feedback” en Feedback for Learning, vol. 70, issue 1 , p. 10-16. 